Recibido: 16-06-2015 Aceptado: 08-07-2015

Palabras clave: gestión de la información, y proceso de investigación. Keywords: information management, research process.

Julyssa Llave Zañartu UAP julussallz@yahoo.es

\section{La gestión de la información y el proceso de investigación en los alumnos de IV ciclo de una Universidad privada de Lima Metropolitana}

\section{Information management and research process in iv term students from a private university of metropolitan Lima}

\author{
Julyssa LLave Zañartu
}

RESUMEN. Se pretende determinar la problemática existente entre la Gestion de la Información y el Proceso de Investigación correspondiente a los alumnos de IV ciclo de una Universidad Privada de Lima. La investigación es explicativa y descriptiva, no experimental, transversal y correlacional. Se analizó cada una de las variables, el tipo de relación existente, así como también se identificó las diferencias correspondientes de estas variables en los alumnos.

Los resultadosindican quela Gestión dela Información interactúa significativamente en el Proceso de Investigación. Así mismo, los alumnos presentan diferencias significativas de acuerdo a los indicadores. Finalmente, los resultados fueron analizados y se construyó un marco doctrinario.

ABSTRACT. This research aims to determine the existing problems for information management and research process among Term Four students at a private university based in Lima. This research is causal and descriptive, non experimental, cross-sectional and correlational. Each variable and the relationship between them were analyzed, and the differences in these variables among the students were identified.

The results indicate that Information Management interacts significantly in the Research Process. In addition, the students show significant differences according to the indicators. Finally, the results were analyzed and a doctrinary framework was then built. 


\section{Introducción}

E n este artículo se presenta un estudio de la Gestión de la Información y Proceso de Investigación en los alumnos de cuarto ciclo de una Universidad Privada de Lima Metropolitana. En esa línea de ideas, se destaca la significatividad de cada variable, en el sentido que los discentes al manipular la información deben tener una cultura informática que les permita ejercer su capacidad selectiva y capturar información con números de estándar de calidad a fin de procesarla a través de un pensamiento creativo y crítico. En esa intencionalidad, se construye el conocimiento y el discente ingresa al proceso de investigación con éxito, elevando, de esta manera, sus niveles de productividad científica.

El objetivo de la investigación fue determinar la problemática entre la Gestión de la Información y Proceso de Investigación en los alumnos de cuarto ciclo de una Universidad privada de Lima de acuerdo a sus dimensiones e indicadores.

El artículo presenta el planteamiento del problema que responde a la siguiente pregunta: ¿¿De qué manera la Gestión de la Información influye en el Proceso de Investigación en los alumnos de IV ciclo de una Universidad Privada de Lima Metropolitana? Se ha tomado como unidad de análisis las encuestas tomadas a alumnos de la Facultad de Administración y Negocios Internacionales de una Universidad privada de Lima. Asimismo, se presenta el marco teórico de cada una de las variables que proporciona el sustento del presente trabajo específicamente, centrado en las doctrinas que constan en el capítulo de Referencias. Finalmente, presenta el sistema de hipótesis y el diseño metodológico propio de una investigación tipo básica, sustantiva. Por el enfoque cuantitativo, es un estudio descriptivo y explicativo se aplica la estadística para la recopilación de los datos y cuenta con una muestra de cincuenta alumnos. Se encontró que los alumnos que gestionan la información transformándola en conocimiento presentan niveles adecuados de productividad científica y comprensión lectora. En tal sentido, mejora tanto su rendimiento como su aprendizaje. Así mismo, se observan diferencias
Alétheia 2015,3 (1) 17 significativas tanto en la gestión de información como el proceso de investigación de los alumnos. En ese orden de ideas, se presentan los resultados, la discusión de los resultados y por último las conclusiones y las respectivas referencias.

\section{Materiales y Método}

\section{Materiales}

El material usado en esta investigación fue:

1. Cuestionario sobre gestión del conocimiento, tecnología de información e innovación.

2. Cuestionario Motivación de Logro: MAPE- 3. Tapia, Montero y Huertas (2000).

\section{Método}

El nivel de investigación es básica y sustantiva porque se encontraron interacciones entre gestión de la información y proceso de investigación en los alumnos de una universidad privada de Lima. Así mismo, se descubrió una relación entre la variables antes mencionadas.

Por el enfoque cuantitativo, el tipo de investigación es un estudio descriptivo-explicativo. Sánchez y Reyes (1985) sostienen que es desscriptivo porque a partir de una muestra probabilística de alumnos de la Escuela de Administración y Negocios Internacionales de una universidad privada, se descubrieron características de las variables materia de estudio y se comprobaron la asociación entre las variables de la población. En la investigación descriptiva se recolecta información relevante en muestras con respecto a un mismo fenómeno. En base a la comparación de los datos recopilados, es posible realizar una comparación entre ellos.

Bernal (2002) señala que la investigación explicativa es la más usada en las Ciencias Sociales. Cuando en un estudio el investigador plantea como objetivo estudiar el porqué de las cosas, hechos, fenómenos o situaciones se trata de investigación explicativa. Cuanto mayor número de variables estén correlacionadas en el estudio y mayor sea la fuerza de las relaciones, más completa será la explicación, según Hernández, Fernández y Baptista (1996). 


\section{UNIFÉ - EPG}

El diseño de la investigación es no experimental, transversal y correlacional, pues esta investigación asocia las variables para establecer comparaciones, teniendo en cuenta los indicadores. Se aplicó los instrumentos en un solo tiempo a efectos de establecer la relación entre las variables.

El procedimiento usado fue el siguiente:

Primero: Determinación de las variables, las dimensiones.

Variable Independiente: Gestión de la Información

Dimensiones:
a) Tecnologia
b) Infraestructura

Variable Dependiente: Proceso de Investigación

Dimensiones:
a) Método
b) Técnicas de recolección de datos
c) Estrategia general de la Investigación

\section{Segundo}

Determinación de sujetos a estudiar:

La investigación tiene una muestra de cincuenta alumnos de la Facultad de Administración y Negocios Internacionales de una Universidad privada de Lima del turno mañana.

\section{Resultados}

Tabla 1

Correlación entre Gestión de la Información y Proceso de Investigación de los alumnos de una Universidad Privada.

\begin{tabular}{lr}
\hline \multicolumn{1}{c}{ Proceso de Investigación } & \multicolumn{1}{c}{ r } \\
\hline Gestión de la Información & \\
Correlación de Pearson & $0.857^{*}$ \\
Significancia bilateral & 0.000 \\
Tamaño de muestra & 50 \\
\hline
\end{tabular}

* La correlación es significativa al nivel 0.01 (bilateral).

La hipótesis general establecía que existe interacción significativa entre Gestión de la Información y Proceso de Investigación de los alumnos de una universidad privada de Lima. Los resultados presentados en la tabla 1 indican una correlación directa positiva y significativa $(\mathrm{r}=0.857$, $\mathrm{p}<0.01)$ entre Gestión de la Información y Proceso de Investigación. En tal sentido, la hipótesis ha sido confirmada.

Tabla 2

Niveles de Gestión de la Información de los alumnos de una universidad Privada.

\begin{tabular}{lcccc}
\hline Niveles & $\begin{array}{r}\text { Total } \\
\text { Porcentaje } \\
\text { de prueba }\end{array}$ & & Estadística \\
\hline Medio & 14 & 28 & & \\
Alto & 36 & 72 & $9.68 *$ & 0.002 \\
Total & 50 & 100 & g.l $=1$ & \\
\hline
\end{tabular}

${ }^{*} \mathrm{p}<0.05$. 1-25: Muy Bajo, 26-50: Bajo, 51-75: Medio, 76-100: Alto

De acuerdo a los resultados, la sub hipótesis que sostenía que los alumnos presentaban diferencias significativas en los niveles de gestión de la información ha sido confirmada y se observan niveles adecuados.

\section{Discusion de resultados}

1. Correlación entre Gestión de la Información $y^{z}$. Proceso de Investigación

Según Escobar, Betancur, Palacioy Dario (2008), gestionar la información constituye una herramienta que facilita el proceso de investigación, pues la gestión involucra el ejercicio de capacidades tanto selectivas como reflexivas a fin de abordar una problemática adecuándose a una metodología de investigación científica. Por esta razón, los autores destacan la significatividad de las variables mencionadas en el artículo de la autora de la siguiente manera:

La capacitación y el entrenamiento de los usuarios de esta tecnología encarnan, entre otros, tres retos principales: El desarrollo del pensamiento espacial, el desarrollo de las competencias en el diseño y aplicación de este instrumento como plataforma de apoyo en el análisis de problemáticas ambientales y de los recursos y, finalmente, la identificación de las estrategias que permiten la articulación de esta tecnología en entornos (Escobar, Betancur, Palacio y Dario, 2008, p. 25). 
Por consiguiente, las explicaciones de los autores demuestran que la gestión de la información incide en el proceso de investigación. La Hipótesis General establecía una fuerte correlación entre las variables: Gestión de la Información y Proceso de Investigación de los alumnos de una universidad privada de Lima. Así, los resultados de la presente investigación confirman la premisa de los autores.

González (2008), en su obra: "Semilletos de Investigación: Una estrategia formativa", destaca un Ptoyecto Educativo que tiene por finalidad formar a los sujetos del proceso educativo para el desarrollo de la ciencia y tecnología en disciplinas humanas. En efecto, el proyecto pedagógico tiene por finalidad difundir una cultura informática, una cultura informativa, una cultura investigativa en el actor educativo con el propósito de elevar sus niveles de productividad científica.

El estudio citado permite afirmar que las variables materia de estudio tienen una correlación alta, lo cual fue evaluado mediante el coeficiente de Correlación de Pearson, que permite analizar el grado de relación existente entre las variables: Gestión de la Información y Proceso de Investigación. En tal sentido, si los alumnos tienen una cultura informática; se repotencia, de esta manera, el proceso de investigación del alumno investigador.

\section{Diferencias significativas en los niveles de Gestión} de la Información.

La gestión de la información constituye un recurso que debe realizarse de manera efectiva; pues solo así se garantiza un feliz proceso de investigación. Al respecto, Rivas (2003), plantea lo siguiente: "la información debe ser vista como otro recurso. El procesamiento de la información; es decir, la clasificación, ordenación, descripción y valotación desde un punto de vista integtal facilita la toma de decisión" (p. 3). En consecuencia, según el autor, el potencial humano gestiona la información, de acuerdo a sus conocimientos, pensamientos, habilidades y competencias. Inchuso destaca la significatividad de la misma cuando afirma que las grandes organizaciones tienen un principal interés: Gestionar la información de una manera más eficiente. En ese orden de ideas, si existen diferencias significativas en los niveles de Gestión de la Información de los alumnos; se debe al propio talento humano universitario, pues se trata de alumnos de cuarto ciclo con culturas informáticas y conocimientos previos adecuados que fortalecen el proceso de investigación. Romero (2002), reafirma lo antetior cuando prescribe que "la información es la materia prima del conocimiento" (p. 425). En consecuencia, las evidencias empíticas confirman la Hupótesis secundaria que establecía diferencias significativas en los niveles de gestión de información. Los resultados fueron validados a través de la Prueba Chi-Cuadrado a fin de analizar las diferencias existentes que muestran el predominio de niveles altos. En efecto, si los niveles de gestión de información son altos, entonces también serán altos lo niveles de productividad cientifica de los alumnos.

\section{Conclusiones}

Euego de realizado el análisis y discusión de los resultados en la presente investigación, se presenta las conclusiones que se desprenden de la importancia de las variables materia de investigación; las mismas que interactúan dentro de una gestión educativa.

1. Existe una correlación alta entre las siguientes variables: Gestión de la Información y Proceso de Investigación. Esto indica que a medida que el alumno gestione la información, sus niveles de productividad científica serán adecuados.

2. Existen diferencias significativas en los niveles de Gestión de la Información, predominando niveles adecuados. En tal sentido, si los niveles de gestión de información son adecuados entonces se garantiza la efectividad en el proceso de investigación de los alumnos investigadores.

\section{Referencias}

Bernal, C. (2000). Metodologia de la Investigación para Administración y Economia. Bogotá, Colombia: Prentice Hall. 
20 UNIFÉ - EPG

Escobar,J., Betancur, T., Palacio, C., y Dario, R (2008). Los retos de la enseñanza de los sistemas de información geográfica integrados a la gestión del medio ambiente y recursos naturales. Gestión y Ambiente, 11,125-136.

González, J. (2008). Semilleros de Investigación: Una estrategia formativa. Psychologia, 2, 185-190.

Hernández. R., Fernández. C., y Baptista, P. (1996). Metodología de la Investigación. México, D. F., México: Mc. Graw Hill

Rivas, J. (2003). La gerencia de información. Biblios, 4, 3-13.

Romero, A. (2002). Las redes de información y su importancia para la investigación científica. Venezolana de Gerencia.19, 425-441.

Sánchez, C., y Reyes, M. (1985). Metodologia y Diseños en la Investigación Cientifica Iima. Perú: Mantaro. 